\title{
THE SPACE OF EXTENSIONS OF A KLEIN SURFACE OF FINITE GENUS
}

\section{A.J. Alonso Gomez and A. Fernandez Arias}

\begin{abstract}
Some of Oikawa's work is extended; the space $P(F)$ of compact extensions of an open unbordered Klein surface of finite genus is introduced. Some properties of compactness and connectedness of $P(F)$ are obtained starting from the compactness and connectedness of $P\left(\tilde{F}_{c}\right)$, $\tilde{F}_{c}$ the complex double of $F$. The uniqueness of the extension is also studied.
\end{abstract}

\section{Introduction}

A compact Riemann (or Klein) surface $W_{0}$ is called a compact extension of the same genus of a Riemann surface (or Klein) surface $F \subset W_{0}$ if $W_{0} \backslash F$ is a union of sets homeomorphic to a set containing one point or to the closed unit disk in the complex plane. (For the notion of genus of a topological surface see W.S. Massey [8], Chapter 1.)

Let us now assume that $F$ and $W_{0}$ are fixed and $W_{0}$ is an extension of $F$ in this sense. We may study extensions in a more general context. An extension of $F$ is a pair $(W, f)$ where $f: F \rightarrow f(W) \nsubseteq W$ is a holomorphic (dianalytic) injection in the above sense.

In $1957 \mathrm{~K}$. Oikawa [9] defined the space $P(F)$ of compact extensions of an open Riemann surface $F$ of finite genus $g>1$ and proved that $P(F)$ is a compact and connected set of the Teichmüller space $T_{g}$. This result generalizes a theorem of $\mathrm{M}$. Heins concerning the moduli of the tori to which a fixed open Riemann surface of genus one can be extended.

In this paper we shall define and study the corresponding space $P(F)$ for an open unbordered non-orientable Klein surface $F$ of finite genus $g>2$.

In Section 2 we give an account of some basic facts on Klein surfaces and Teichmüller spaces of Klein surfaces. The standard references are N.L. Alling and N. Greenleaf [2] and M. Seppälä [11].

In Section 3 we introduce the space $P(F)$ of compact extensions of an open unbordered non-orientable Klein surface $F$ of finite genus $g>2$. We prove that the standard isometry

$$
i: T_{g}\left(W_{0}\right) \rightarrow T_{g-1}\left(\tilde{W}_{0 c}\right)
$$

1980 Mathematics Subject Classification: 30F99 Riemann surfaces. Key words: Klein surface, dianalytic structure, dianalytic morphism.

This work was partially supported by DGICYT Grant PR 84-0338. 
from the Teichmüller space of Klein surfaces into the Teichmüller space of Riemann surfaces sends $P(F)$ into $P\left(\tilde{F}_{c}\right), \tilde{F}_{c}$ the complex double of $F$, and derive in Sections 4-5 the relative compactness and connectedness of $P(F)$.

Finally, Section 6 is dedicated to the study of the uniqueness of the extension of a Klein surface.

\section{Preliminaries}

2.1. Klein surfaces. 2.1.1. Let $W$ be a topological, possibly bordered, surface. We call an atlas $A=\left\{\left(U_{i}, z_{i}\right) \mid i \in I\right\}$ of $W$ dianalytic if the coordinate transition maps $z_{i} \circ z_{j}^{-1}$ belong to the class $C^{2}$ and satisfy either

$$
\frac{\partial\left(z_{i} \circ z_{j}\right)^{-1}}{\partial \bar{z}}=0 \quad \text { or } \quad \frac{\partial\left(z_{i} \circ z_{j}\right)^{-1}}{\partial z}=0
$$

in a neighbourhood of every interior point of $z_{j}\left(U_{i} \cap U_{j}\right)$ in the complex plane $\mathbf{C}$. Two dianalytic atlases $A_{1}, A_{2}$ are called equivalent if $A_{1} \cup A_{2}$ is a dianalytic atlas. An equivalence class $\bar{A}$ of dianalytic atlases of $W$ is called a dianalytic structure of $W$. The pair $(W, \bar{A})$ is called a Klein surface. Usually, we shall abbreviate and just write $W$.

A Klein surface need not be orientable. On the other hand, every Riemann surface has a natural dianalytic structure and can be regarded as an orientable Klein surface.

In this study we shall only be interested in the unbordered Klein surfaces. Throughout the paper we shall use the notation UKS to denote the unbordered Klein surfaces and UNOKS to denote the unbordered non-orientable Klein surfaces.

Let $f: W_{1} \rightarrow W_{2}$ be a continuous mapping between two UKS. We call $f$ a morphism if for each $p \in W_{1}$ and a local variable $z$ at $p$ there is a local variable $w$ at $f(p)$ such that we can find an analytic function $F$ for which the relationship $w \circ f=F \circ z$ holds, in some neighbourhood of $p$. A morphism is an isomorphism if it is a homeomorphism.

2.1.2. A useful technique for translating problems on Klein surfaces into problems on Riemann surfaces is yielded by the existence of the complex double of a Klein surface.

The complex double of a Klein surface $W$ is a triple $\left(\tilde{W}_{c}, \varrho, \sigma\right)$ satisfying the following conditions:

i) $\tilde{W}_{c}$ is a Riemann surface;

ii) the morphism $\varrho: \tilde{W}_{c} \rightarrow W$ is a double cover;

iii) $\sigma: \tilde{W}_{c} \rightarrow \tilde{W}_{c}$ is an antianalytic involution satisfying $p \circ \sigma=p$.

One can give an explicit construction of such a triple (see N.L. Alling and N. Greenleaf [2], p. 37). The complex double is unique modulo isomorphisms and 
$\tilde{W}_{c}$ is disconnected if and only if $W$ is unbordered and orientable; in such a case $\tilde{W}_{c}$ consists of two sheets identical to $W$.

For a compact Klein surface $W$ with $k$ boundary componenents we have the following relationship between the genus $g$ of $W$ and $g_{c}$ of $\tilde{W}_{0 c}$ :

$$
g_{c}= \begin{cases}2 g+k-1 & \text { if } w \text { is orientable } \\ g+k-1 & \text { if } W \text { is non-orientable }\end{cases}
$$

We shall mainly be concerned with the case of unbordered non-orientable compact $W$ 's, in which case $g_{c}=g-1$ holds.

2.1.3. Let now $W$ be a UKS. A meromorphic function $f_{A}$ relative to an atlas $A=\left\{\left(U_{i}, z_{i}\right) \mid i \in I\right\}$ of $W$ is a collection $\left\{f_{i}\right\}_{i \in I}$ of maps $f_{i}: U_{i} \rightarrow \mathbf{C}$, where $\mathbf{C}$ denotes the Riemann sphere, satisfying the following conditions:

i) The functions $f_{i} \circ z_{i}^{-1}$ are meromorphic for every $i \in I$;

ii) if $p \in U_{i} \cap U_{j}$, then $f_{i}(p)=f_{j}(p)$ when $z_{i} \circ z_{j}^{-1}$ is analytic and $f_{i}(p)=\overline{f_{j}(p)}$ otherwise.

If $f_{A_{1}}$ and $f_{A_{2}}$ are meromorphic functions relative to $A_{1}, A_{2}$, respectively, we shall say that they are equivalent if $f_{A_{1}} \cup f_{A_{2}}$ is a meromorphic function relative to $A_{1} \cup A_{2}$. An equivalence class $\underline{f}$ of meromorphic functions relative to dianalytic atlases of $W$ will be called a meromorphic function on $W$.

Meromorphic functions on $W$ form a field $E(W)$. This field contains a copy of $\mathbf{C}$ if and only if $W$ is a Riemann surface.

Meromorphic functions are not maps; yet we can associate with $\underline{f} \in E(W)$ a map $f: W \rightarrow \bar{H}$, where $\bar{H}$ is the closed upper half plane with the point at infinity. In fact, if we write $f_{j}=a_{j}+i b_{j}$, the maps $\left\{a_{j}+i\left|b_{j}\right|\right\}_{j \in J}$ can be patched together to form a map $f$ on $W$ which will be continuous except at the poles of $\underline{f}$ and such that its real part is harmonic at the points of continuity.

Given a non-constant morphism $f: W_{1} \rightarrow W_{2}$ between UKS, there is a unique $\mathbf{R}$-monomorphism $f^{*}: E\left(W_{2}\right) \rightarrow E\left(W_{1}\right)$ with the property that the map of $f^{*} g$ is $g \circ f$, where $g$ is the map of $g$ for all $g \in E\left(W_{2}\right)$.

Let now $\varrho: \tilde{W}_{c} \rightarrow W$ be the double cover associated to the complex double of a UNOKS $W$. Then $\varrho^{*}(E(W))$ is the fixed field of $E\left(\tilde{W}_{c}\right)$ under $\sigma^{*}$ and $E\left(\tilde{W}_{c}\right)=\left(\varrho^{*}(E(W))\right)(\sqrt{-1})$.

2.2. Teichmüller spaces of Klein surfaces. 2.2.1. Let $f: W_{1} \rightarrow W_{2}$ be a homeomorphism between UNOKS $W_{1}, W_{2}$. We call $f K$-quasiconformal, for a finite $K \geq 1$, if for each $p \in W$ and for each local $z$ at $p$ we can choose a local variable $w$ at $f(p)$ such that the mapping $w \circ f \circ z^{-1}$ is $K$-quasiconformal in a neighbourhood of $z(p)$. We shall say that $f$ is quasiconformal if it is $K$ quasiconformal for some finite $K$.

Let now $W_{0}$ be a fixed compact UKS of genus $g \geq 2$ and consider pairs $(W, f)$ where $f: W_{0} \rightarrow W$ is a quasiconformal homeomorphism. We shall say that two 
pairs $\left(W_{1}, f_{1}\right),\left(W_{2}, f_{2}\right)$ are equivalent if there exists a dianalytic isomorphism $L: W_{1} \rightarrow W_{2}$ homotopic to $f_{2} \circ f_{1}^{-1}$. The set of all equivalence classes $\langle W, f\rangle$ is the Teichmüller space $T_{g}\left(W_{0}\right)$. The space $T_{g}\left(W_{0}\right)$ is a metric space endowed with the distance

$$
d\left(\left\langle W_{1}, f_{1}\right\rangle,\left\langle W_{2}, f_{2}\right\rangle\right)=\frac{1}{2} \inf _{L}\left\{\log K_{L}\right\},
$$

where $L$ ranges in the class of all quasiconformal mappings in the homotopy class of $f_{2} \circ f_{1}^{-1}$ and $K_{L}$ denotes maximal dilatation.

The metric space $T_{g}\left(W_{0}\right)$ for a UKS $W_{0}$ is homeomorphic to a simply connected open set of $\mathrm{C}^{3 g-3}$ in the orientable case, i.e., in the case of Riemann surfaces and of $\mathbf{R}^{3 g-3}$ in the non-orientable case, that is in the case of UNOKS.

The Teichmüller spaces $T_{g}\left(W_{0}\right)$ and $T_{g}(W)$ of two compact orientable UKS, i.e., Riemann surfacs, of the same genus, are isometrically bijective, therefore we can talk of the Teichmüller space $T_{g}$ of Riemann surfaces of genus $g$. Also the Teichmüller spaces of compact UNOKS of the same genus are isometrically bijective and we speak of the Teichmüller space $T_{g}$ of UNOKS.

2.2.2. Let $W_{0}$ be a compact UNOKS of genus $g \geq 2$ and $\tilde{W}_{0 c}$ its complex double which will have genus $g-1$. An isometry

$$
i: T_{g}\left(W_{0}\right) \rightarrow T_{g-1}\left(\tilde{W}_{0 c}\right)
$$

from the Teichmüller space of UNOKS of genus $g$ into the Teichmüller space of Riemann surfaces of genus $g-1$ is defined in a natural way; we shall call it the canonical isometry. In fact, if $f_{1}: W_{0} \rightarrow W_{1}$ is a quasiconformal mapping of UNOKS, we can lift it in a unique way to a quasiconformal mapping $\tilde{f}_{1}: \tilde{W}_{0 c} \rightarrow$ $\tilde{W}_{1 c}$, and if $f_{2}: W_{0} \rightarrow W_{2}$ is another quasiconformal mapping of UNOKS and $\tilde{f}_{2}: \tilde{W}_{0 c} \rightarrow \tilde{W}_{2 c}$ the corresponding lifting, then if $f_{2} \circ f_{1}^{-1}$ is homotopic to a dianalytic isomorphism $L: W_{1} \rightarrow W_{2}$, we can lift the homotopy to conclude that $\tilde{f}_{2} \circ \tilde{f}_{1}^{-1}$ is homotopic to a conformal map $\tilde{L}: \tilde{W}_{1 c} \rightarrow \tilde{W}_{2 c}$, so that we have a well-defined mapping $i: T_{g}\left(W_{0}\right) \rightarrow T_{g-1}\left(\tilde{W}_{0 c}\right)$ by setting $i(\langle W, f\rangle)=\left\langle\tilde{W}_{c}, \tilde{f}\right\rangle$. It is proved that this mapping is in fact an isometry (see [11]).

\section{The space $P(F)$}

We shall denote open (non-compact) UKS by $F$ and compact ones by $W$.

Let $F$ be an open UNOKS of finite genus $g>2$ and $f: F \rightarrow f(F) \nsubseteq W$ a compact extension of the same genus as $F$. Then $f$ induces an homomorphism $f^{*}: \pi_{1}(F) \rightarrow \pi_{1}(W)$ from the fundamental group $\pi_{1}(F)$ of $F$ into the fundamental group $\pi_{1}(W)$ of $W$.

Lemma 3.1. The following assertions hold:

i) $f^{*}: \pi_{1}(F) \rightarrow \pi_{1}(W)$ is onto;

ii) $\operatorname{Ker}\left(f^{*}\right)$ depends only upon $F$, i.e., if $f_{1}: F \rightarrow f_{1}(F) \nsubseteq W_{1}$ is another extension with $W_{1}$ compact of the same genus as $F$, then $\operatorname{Ker}\left(f^{*}\right)=\operatorname{Ker}\left(f_{1}^{*}\right)$. 
The content of Lemma 3.1 is quite intuitive. The check i) we observe that the image $f(F)$ is a subsurface of $W$ of the same genus of $F$ and hence of $W$. Therefore we can find a set of generators of $\pi_{1}(W)$ in $f(F)$ and that implies that $f^{*}$ is onto. On the other hand, ii) can be seen by observing that $\operatorname{Ker}\left(f^{*}\right)$ is generated by those homotopy classes corresponding to curves in $F$ which are homotopic in $W_{0}$ to a boundary component of $F$. In other words, the kernel $\operatorname{Ker}(f)$ can be described as the smallest subgroup of $\pi_{1}(F)$ containing every closed Jordan curve which divides $F$ into two parts, one of which is planar.

Let now $F$ be an open UNOKS of genus $g, g>2$ and consider the family $\{(W, f)\}$ of all extensions where $W$ is compact and of the same genus as $F$. It is clear that the $W$ 's must also be non-orientable, i.e., all of them are compact UNOKS of genus $g$.

Let us denote this family by $B(F)$. By [3], we have $B(F) \neq \emptyset$.

Let $\left(W_{0}, f_{0}\right)$ be a fixed extension in $B(F)$. For any $(W, f)$ the mapping $f \circ f_{0}^{-1}$ maps $f_{0}(F) \subset W_{0}$ isomorphically into $W$; then by Lemma 3.1, it induces a homomorphism

$$
\left(f \circ f_{0}^{-1}\right)^{*}: \pi_{1}\left(f_{0}(F)\right) \rightarrow \pi_{1}(W)
$$

and therefore an isomorphism

$$
\alpha_{f}: \pi_{1}\left(W_{0}\right)=\frac{\pi_{1}\left(f_{0}(F)\right)}{\operatorname{Ker}\left(f \circ f_{0}^{-1}\right)^{*}} \rightarrow \pi_{1}(W) .
$$

By Nielsen's theorem (see [13], Chapter 5) $\alpha_{f}$ is induced by a homeomorphism $h: W_{0} \rightarrow W$, and it is wellknown that it can be taken quasiconformal. We conclude that a compact extension $(W, f)$ determines a point $\langle W, h\rangle$ of the Teichmüller space $T_{g}\left(W_{0}\right)$, i.e., we have a map

$$
B(F) \ni(W, f) \rightarrow\langle W, h\rangle \in T_{g}\left(W_{0}\right) .
$$

Given an extension $(W, f)$ in $B(F)$ and a point $\langle W, h\rangle$ of $T_{g}\left(W_{0}\right),\langle W, h\rangle$ corresponds to $(W, f)$ if and only if $h_{\mid f_{0}(F)}$ is homotopic to $f$.

The image of $B(F)$ in $T_{g}\left(W_{0}\right)$ by this map will be denoted by $P(F)$.

\section{Boundedness and relative compactness of $P(F)$}

In this section we deduce the boundedness and the relative compactness of $P(F), F$ a UNOKS, from the boundedness and compactness of $P\left(\tilde{F}_{c}\right), \tilde{F}_{c}$ the complex double of $F$, making use of the canonical isometry $i: T_{g}\left(W_{0}\right) \rightarrow$ $T_{g-1}\left(\tilde{W}_{0 c}\right)$.

From now on we shall identify $F$ with $f_{0}(F)$ so that we shall regard $F$ as a subset of $W_{0}$.

Given an extension $(W, f)$ of $F$ of UNOKS, we can lift $f$ in a unique way to a conformal mapping $\tilde{f}$ to obtain an extension $\left(\tilde{W}_{c}, \tilde{f}\right)$ of $\tilde{F}_{c}$ of Riemann surfaces (see A. Fernandez [4]). 
Theorem 4.1. The canonical isometry $i: T_{g}\left(W_{0}\right) \rightarrow T_{g-1}\left(\tilde{W}_{0 c}\right)$ sends $P(F)$ into $P\left(\tilde{F}_{c}\right)$. Given $\left\langle W, h_{f}\right\rangle \in P(F)$ corresponding to the compact extension $(W, f)$ of $F, i\left(\left\langle W, h_{f}\right\rangle\right)=\left\langle\tilde{W}_{c}, \tilde{h}_{\tilde{f}}\right\rangle$, where $\left\langle\tilde{W}_{c}, \tilde{h}_{\tilde{f}}\right\rangle$ is the point of $P\left(\tilde{F}_{c}\right)$ corresponding to the compact extension $\left(\tilde{W}_{c}, \tilde{f}\right)$ of $\tilde{F}_{c}$.

Corollary 4.1. The set $P(F) \subset T_{g}$ is a relatively compact set and therefore a bounded set.

Proof of Theorem 4.1. Let $\left\langle W, h_{f}\right\rangle \in P(F)$ correspond to the compact extension $(W, f)$ of $F$. Then $h_{f}$ can be lifted in a unique way to a quasiconformal mapping $\tilde{h}: \tilde{W}_{0 c} \rightarrow \tilde{W}_{c}$. By the definition of the canonical isometry we have $i\left(\left\langle W, h_{f}\right\rangle\right)=\left\langle\tilde{W}_{c}, \tilde{h}\right\rangle$. Therefore the statement of Theorem 4.1 is $\left\langle\tilde{W}_{c}, \tilde{h}\right\rangle=\left\langle\tilde{W}_{c}, \tilde{h}_{\tilde{f}}\right\rangle$.

In fact, $f$ and $h_{f \mid F}$ are homotopic and this homotopy can be lifted to a homotopy between $\tilde{f}$ and $\tilde{h}_{\tilde{F}_{c}}$, but this implies that $\tilde{f}, \tilde{h}_{\tilde{f}}, \tilde{h}$ all induce the same group isomorphism from $\pi_{1}\left(\tilde{W}_{0 c}\right)$ to $\pi_{1}\left(\tilde{W}_{c}\right)$ and as a consequence $\left\langle\tilde{W}_{c}, \tilde{h}_{\tilde{f}}\right\rangle=$ $\left\langle\tilde{W}_{c}, \tilde{h}\right\rangle$. This completes the proof of Theorem 4.1.

Proof of Corollary 4.1. We prove the relative compactness of $i(P(F))$ in $i\left(T_{g}\left(W_{0}\right)\right)$. By Theorem 4.1 we have the relationship

$$
i(P(F)) \subset P\left(\tilde{F}_{c}\right) \cap i\left(T_{g}\left(W_{0}\right)\right) .
$$

To prove the relative compactness of $i(P(F))$ in $i\left(T_{g}\left(W_{0}\right)\right)$, we take an arbitrary sequence $\left\{\left\langle\tilde{W}_{c n}, \tilde{h}_{n}\right\rangle\right\}_{n \in \mathbf{N}}$ in $i(P(F))$ and show that this sequence has a convergent subsequence in $i\left(T_{g}\left(W_{0}\right)\right)$.

In fact, since the sequence $\left\{\left\langle\tilde{W}_{c n}, \tilde{h}_{n}\right\rangle\right\}_{n \in \mathbf{N}}$ is in the compact set $P\left(\tilde{F}_{c}\right)$, there is a subsequence $\left\{\left\langle\tilde{W}_{c n_{k}}, \tilde{h}_{n_{k}}\right\rangle\right\}_{k \in \mathbf{N}}$ converging to an element of $P\left(\tilde{F}_{c}\right) \subset$ $T_{g-1}\left(\tilde{W}_{0 c}\right)$. Therefore the subsequence $\left\{\left\langle\tilde{W}_{c n_{k}}, \tilde{h}_{n_{k}}\right\rangle\right\}_{k \in \mathbf{N}}$ is a Cauchy sequence in $i\left(T_{g}\left(W_{0}\right)\right)$, which is complete, i.e., it converges in $i\left(T_{g}\left(W_{0}\right)\right)$, q.e.d.

\section{Connectedness of $P(F)$}

We recall the relationship

$$
i(P(F)) \subset P\left(\tilde{F}_{c}\right) \cap i\left(T_{g}\left(W_{0}\right)\right) \subset T_{g-1}\left(\tilde{W}_{0 c}\right) .
$$

K. Oikawa [9] (see also T. Jussila [5]) proved that $P\left(\tilde{F}_{c}\right)$ is connected showing that an arbitrary point $\left\langle\tilde{W}_{0 c}, \tilde{h}\right\rangle$ in $P\left(\tilde{F}_{c}\right)$ can be connected to the point $\left\langle\tilde{W}_{0 c}, \tilde{I}\right\rangle$ of $P\left(\tilde{F}_{c}\right)$ by a path contained in $P\left(\tilde{F}_{c}\right)$.

Here we prove the connectedness of $P(F)$, or equivalently that of $i(P(F))$, in the case where the boundary of $F$ is formed by a finite number of closed analytic curves. To do this we check that the path constructed by K. Oikawa joining a point $\left\langle\tilde{W}_{0 c}, \tilde{h}\right\rangle \in i(P(F)) \subset P\left(\tilde{F}_{c}\right)$ to $\left\langle\tilde{W}_{0 c}, \tilde{I}\right\rangle=i\left(\left\langle W_{0}, I\right\rangle\right)$ is entirely contained in $i(P(F))$. 
Theorem 5.1. In the above mentioned conditions $P(F)$ is connected.

Proof. We shall first consider the case where $F$ is a subdomain of $W_{0}$ such that its boundary consists of one single component $C$. Then $\tilde{F}_{c}$ will be a subdomain of $\tilde{W}_{0 c}$ whose boundary will be formed by two components $C_{1}, C_{2}$ such that $\sigma\left(C_{1}\right)=C_{2}$.

Let $\left\langle\tilde{W}_{c}, \tilde{h}\right\rangle=i(\langle W, h\rangle) \in i(P(F))$ and $(W, f)$ an extension of $F$ with $h_{\mid F} \simeq$ $f$. Next we describe the path constructed by K. Oikawa in this particular case.

We take a ring domain $A$ in $F$, bounded by $C=\partial F$ and an analytic curve. In $\tilde{F}_{c}$ we shall have two ring domains $A_{1}, A_{2}$ such that $\sigma\left(A_{1}\right)=A_{2}$, the boundary of each of them being formed by one analytic curve and the corresponding $C_{i}$. Since the domains $W_{0} \backslash(F \backslash A)$ and $W \backslash f(F \backslash A)$ are simply connected, we can map them onto the unit disc by mappings $\xi_{0}, \xi$, respectively, in such a way that the counterimages of the origins by $\xi_{0}, \xi$ are in $W_{0} \backslash F, W \backslash f(F)$, respectively. The composed function $\xi \circ f \circ \xi_{0}^{-1}$ maps the ring domain $\xi_{0}(A)$ onto $\xi(f(A))$ sending the unit circle onto itself.

We can find a family of functions $\Phi_{t}, 0 \leq t \leq 1$, satisfying the following conditions (see K. Oikawa [9]):

i) $\Phi_{t}$ is a univalent analytic function of $\xi_{0}(A)$ into the origin-punctured unit disc, mapping the unit circle onto itself. Let $\Delta_{t}$ be the image unit discs;

ii) for every $z$, the mapping $t \rightarrow \Phi_{t}(z)$ is continuous;

iii) $\Phi_{t=0}(z) \equiv z$

iv) $\Phi_{t=1}(z)=\xi \circ f \circ \xi_{0}(z)$, for each $z \in \xi_{0}(A)$.

For each $t$ we consider two copies $\Delta_{t}^{1}, \Delta_{t}^{2}$ of $\Delta_{t}$, and we construct a Riemann surface $\tilde{W}_{t}$ as follows:

$$
\tilde{W}_{t}=\tilde{F}_{c} \cup \Delta_{t}^{1} \cup \Delta_{t}^{2} / \sim
$$

where $\sim$ means identification of the points $p$ of $A_{1}$ and $A_{2}$ with the points of $\Delta_{t}^{1}$ and $\Delta_{t}^{2}$, respectively, corresponding to the point $\Phi_{t} \circ \xi_{0} \circ \varrho_{0}(p), \varrho_{0}: \tilde{W}_{0 c} \rightarrow W_{0}$ the projection associated to the complex double of $W_{0}$. The quotient $\tilde{W}_{t}$ carries the quotient topology and the conformal structure is defined in the natural way starting from the conformal structure of $\tilde{F}_{c}$ and that of $\Delta_{t}^{1}$ and $\Delta_{t}^{2}$.

In $\tilde{W}_{t}$, an antianalytic involution $\sigma_{t}$ is defined in a natural way. Let $\Pi: \tilde{F}_{c} \cup$ $\Delta_{t}^{1} \cup \Delta_{t}^{2} \rightarrow W_{t}$ be the canonical projection; then if $\bar{p} \in \Pi\left(\tilde{F}_{c}\right)$, say $\bar{p}=\Pi(p)$, we set $\sigma_{t}(\bar{p})=\Pi \circ \sigma(p)$, where $\sigma$ is the antianalytic involution of $\tilde{W}_{0 c}$. If $\bar{p} \in \Pi\left(\Delta_{t}^{1}\right)$, say $\bar{p}=\Pi\left(w^{1}\right), w^{1} \in \Delta_{t}^{1}$ then $\sigma_{t}(\bar{p})=\Pi\left(w^{2}\right), w^{2} \in \Delta_{t}^{2}$, where $w^{1}, w^{2}$ correspond to the same point of $\Delta_{t}$.

A quasiconformal mapping

$$
H_{t}^{1}: \xi_{0}\left(W_{0} \backslash(F \backslash A)\right) \rightarrow \Delta_{t}^{1}
$$

is defined by

$$
H_{t}^{1}\left(r e^{i \theta}\right)=r \Phi_{t}\left(e^{i \theta}\right), \quad 0 \leq r \leq 1, \quad 0 \leq \theta \leq 2 \pi
$$


We define a quasiconformal mapping $\tilde{h}_{t}$ from $\tilde{W}_{0 c}$ onto $\tilde{W}_{t}$ by

$$
\tilde{h}_{t}= \begin{cases}\Pi \circ \tilde{I} & \text { in } \tilde{F}_{c} \backslash A_{1} \cup A_{2} \\ \Pi \circ H_{t}^{1} \circ \xi_{0} \circ \varrho_{0} & \text { in } \tilde{W}_{0 c} \backslash\left(F_{c} \backslash A_{1}\right) \\ \sigma \circ \Pi \circ H_{t}^{1} \circ \xi_{0} \circ \varrho_{0} & \text { in } \tilde{W}_{0 c} \backslash\left(F_{c} \backslash A_{2}\right)\end{cases}
$$

and a conformal mapping $\tilde{f}_{t}$ from $\tilde{F}_{c}$ into $\tilde{W}_{t}$ by $\tilde{f}_{t}=\Pi \circ \tilde{I}$.

Then $\left\langle\tilde{W}_{t}, \tilde{h}_{t}\right\rangle, 0 \leq t \leq 1$, is a continuous path in $P\left(\tilde{F}_{c}\right)$ joining $\left\langle\tilde{W}_{0 c}, \tilde{I}\right\rangle$ and $\left\langle\tilde{W}_{c}, \tilde{h}\right\rangle$ (see Jussila [5]). Finally, we observe that $\left\langle\tilde{W}_{t}, \tilde{h}_{t}\right\rangle \in i(P(F))$; in fact,

$$
\left\langle\tilde{W}_{t}, \tilde{h}_{t}\right\rangle=i\left(\left\langle\tilde{W}_{t} /_{\sigma_{t}}, \tilde{h}_{t}\right\rangle\right)
$$

where

$$
h_{t}: W_{0} \rightarrow W_{t}=\tilde{W}_{t} /_{\sigma_{t}}
$$

is the projection of $\tilde{h}_{t}$, which makes sense since

$$
\tilde{h}_{t}(\sigma(p))=\sigma_{t}\left(\tilde{h}_{t}(p)\right), \quad p \in \tilde{W}_{0 c} .
$$

Finally, if the boundaty of $F$ consists of $n>1$ components, the boundary of $\tilde{F}_{c}$ will consist of $2 n$ components which will be pairwise symmetric. Then we have to take a ring domain around each of the boundary components and proceed as above for every ring domain.

\section{Uniqueness of extensions}

6.1. In [10], K. Oikawa proved the following result:

Theorem A. Let $F$ be an open Riemann surface such that $F \in O_{A D}$. Then $P(F)$ consists of a single point. If $F \notin O_{A D}$, then $P(F)$ contains an interior point.

We recall that the class $O_{A D}$ is the class of Riemann surfaces which do not carry a non-constant analytic function with finite Dirichlet integral. In [1], Chapter IV, one can find a treatment of this and other classes of Riemann surfaces.

As a consequence of Theorem A, Oikawa got the following result.

Theorem B. Let $F$ be an open Riemann surface of finite genus $g \geq 1$. Then all the closed Riemann surfaces of genus $g$ which are extensions of $F$ are conformally equivalent to each other if and only if $F$ is of $O_{A D}$. 
In this section we obtain a version of this result for UNOKS.

6.2. First we define the Dirichlet integral of an analytic function $f$ on a UNOKS $X$.

Let $f=\left\{f_{j}\right\}_{j \in J}$ be an analytic function relative to an atlas $A=$ $\left\{\left(U_{j}, z_{j}\right)\right\}_{j \in J}$ of a UNOKS $X$. Then $f_{j}=u_{j}+i v_{j}, f_{j} \circ z_{j}^{-1}$ is analytic and $u_{j} \circ z_{j}^{-1}$ is harmonic.

Now if $u$ is a harmonic map on a plane domain $\Omega$, the integral

$$
\iint_{\Omega}\left[\left(\frac{\partial u}{\partial x}\right)^{2}+\left(\frac{\partial u}{\partial y}\right)^{2}\right] g d x d y
$$

where $g: \Omega \rightarrow \mathbf{R}$ is a continuous function, is invariant under conformal or anticonformal mappings.

The family $\left\{u_{j}\right\}_{j \in J}$ of real parts of an analytic function on a UNOKS can be patched to form a map $u$ on $X$. We define the Dirichlet integral

$$
\begin{aligned}
D_{X}(f)=D_{X}(u) & =\iint_{X}\left[\left(\frac{\partial u}{\partial x}\right)^{2}+\left(\frac{\partial u}{\partial y}\right)^{2}\right] d x d y \\
& =\sum_{j} \iint_{X}\left[\left(\frac{\partial u}{\partial x}\right)^{2}+\left(\frac{\partial u}{\partial y}\right)^{2}\right] g_{j} d x d y,
\end{aligned}
$$

where $\left\{g_{j}\right\}_{j \in J}$ is a partition of unity subordinated to $\left\{U_{j}\right\}_{j \in J}$ and where

$$
\iint_{X}\left[\left(\frac{\partial u}{\partial x}\right)^{2}+\left(\frac{\partial u}{\partial y}\right)^{2}\right] g_{j} d x d y
$$

stands for

$$
\int_{z_{j}} \int_{\left(U_{j}\right)}\left[\left(\frac{\partial\left(u \circ z_{j}^{-1}\right)}{\partial x}\right)^{2}+\left(\frac{\partial\left(u \circ z_{j}^{-1}\right)}{\partial y}\right)^{2}\right]\left(g_{j} \circ z_{j}^{-1}\right) d x d y .
$$

The existence of partitions of unity is ensured by the fact that UNOKS have a countable basis of open sets and it is easy to check that the above definition does not depend on the particular partition of unity chosen.

We define $O_{A D}^{K}$ to be the class of all UNOKS which do not carry non-constant analytic functions with finite Dirichlet integral.

Theorem 6.1. $X$ belongs to $O_{A D}^{K}$ if and only if $\tilde{X}_{c}$ belongs to $O_{A D}$.

Proof. Let $f$ be an analytic function with finite Dirichlet integral and $u$ the real map obtained in the way described above.

The projection $\varrho: \tilde{X}_{c} \rightarrow X$ is a dianalytic morphism. Let then $\varrho^{*}: E(X) \rightarrow$ $E\left(\tilde{X}_{c}\right)$ be the $\mathbf{R}$-monomorphism associated to $\varrho$. The real part $u^{*}$ of $\varrho^{*}(f)$ is obtained, by the definition of $\varrho^{*}$, by lifting $u$ to $\tilde{X}_{c}$. 
Now, given a chart $(U, z)$ at a point $x \in X$ and a continuous function $g: U \rightarrow$ $\mathbf{R}$, let $\left(p^{-1}(U)=\tilde{U}, \tilde{z}\right)$ be the chart in $\tilde{X}_{c}$ associated with $(U, z)$ in the standard way and $\tilde{g}: \tilde{U} \rightarrow \mathbf{R}$ the continuous function obtained from $g$ by lifting to $\tilde{U}$. Then we have

$$
\begin{aligned}
& \iint_{\tilde{U}}\left[\left(\frac{\partial u^{*}}{\partial x}\right)^{2}+\left(\frac{\partial u^{*}}{\partial y}\right)^{2}\right] \tilde{g} d x d y \\
& =2 \iint_{U}\left[\left(\frac{\partial u}{\partial x}\right)^{2}+\left(\frac{\partial u}{\partial y}\right)^{2}\right] g d x d y
\end{aligned}
$$

If $\left\{g_{j}\right\}_{j \in J}$ is a partition of unity in $X$ subordinated to a covering $\left\{U_{j}\right\}_{j \in J}$ of $X$, where we shall assume $U_{j}$ to be simply connected for every $j \in J$, we obtain a partition of unity $\left\{\tilde{g}_{j}\right\}_{j \in J}$ in $\tilde{X}_{c}$ subordinated to the covering $\left\{\tilde{U}_{j}\right\}_{j \in J}$ by lifting the $g_{j}$ to the $\tilde{U}_{j}$.

We deduce from (1)

$$
\begin{aligned}
D_{\tilde{X}_{c}}\left(\varrho^{*}(f)\right) & =D_{\tilde{X}_{c}}\left(u^{*}\right) \\
& =\sum_{j} \iint_{\tilde{U}_{j}}\left[\left(\frac{\partial u^{*}}{\partial x}\right)^{2}+\left(\frac{\partial u^{*}}{\partial y}\right)^{2}\right] \tilde{g}_{j} d x d y \\
& =2 \sum_{j} \iint_{U_{j}}\left[\left(\frac{\partial u}{\partial x}\right)^{2}+\left(\frac{\partial u}{\partial y}\right)^{2}\right] g_{j} d x d y \\
& =2 D_{X}(u)=2 D_{X}(f) .
\end{aligned}
$$

We conclude that $\varrho^{*}(f)$ is an analytic function on $\tilde{X}_{c}$ with finite Dirichlet integral. This completes the first part of the proof.

Conversely, let us assume that $\tilde{X}_{c}$ carries a non-constant analytic function $\tilde{f}$ with finite Dirichlet integral. We can decompose $\tilde{f}$ into the form

$$
\tilde{f}=\tilde{f}_{1}+i \tilde{f}_{2}
$$

where

$$
\tilde{f}_{1}=\frac{\left(\tilde{f}+\sigma^{*}(\tilde{f})\right)}{2}, \quad \tilde{f}_{2}=\frac{\left(\tilde{f}-\sigma^{*}(\tilde{f})\right)}{2 i} .
$$

Since $\sigma^{*}\left(\tilde{f}_{1}\right)=\tilde{f}_{1}, \sigma^{*}\left(\tilde{f}_{2}\right)=\tilde{f}_{2}, \tilde{f}_{1}, \tilde{f}_{2} \in \varrho^{*}(E(X))$ (see N.L. Alling and N. Greenleaf [2], p. 41). Let us consider one of them, say $\tilde{f}_{1}$; then there will be $f_{1} \in E(X)$ such that $\varrho^{*}\left(f_{1}\right)=\tilde{f}_{1}$.

On the other hand, if $U$ is a simply connected open set of $X$ and $\tilde{U}=p^{-1}(U)$, we have the relationship

$$
(f \circ \sigma)_{\mid \tilde{U}}=\kappa \circ \sigma^{*}(f)_{\mid \tilde{U}}
$$


where $\kappa$ denotes complex conjugation, and since the integral

$$
\iint_{\tilde{U}}\left[\left(\frac{\partial \tilde{u}}{\partial x}\right)^{2}+\left(\frac{\partial \tilde{u}}{\partial y}\right)^{2}\right] \tilde{g} d x d y
$$

is invariant under conformal and anticonformal mappings, where $\tilde{u}=\operatorname{Re} \tilde{f}$ and $\tilde{g}: \tilde{U} \rightarrow \mathbf{R}$ is a continuous function, we conclude

$$
\begin{aligned}
& \iint_{\tilde{U}}\left[\left(\frac{\partial \tilde{u}}{\partial x}\right)^{2}+\left(\frac{\partial \tilde{u}}{\partial y}\right)^{2}\right] \tilde{g} d x d y \\
& =\iint_{\tilde{U}}\left[\left(\frac{\partial(\tilde{u} \circ \sigma)}{\partial x}\right)^{2}+\left(\frac{\partial(\tilde{u} \circ \sigma)}{\partial y}\right)^{2}\right](\tilde{g} \circ \sigma) d x d y \\
& =\iint_{U}\left[\left(\frac{\partial\left(\operatorname{Re}\left(\sigma^{*}(\tilde{f})\right)\right)}{\partial x}\right)^{2}+\left(\frac{\partial\left(\operatorname{Re}\left(\sigma^{*}(\tilde{f})\right)\right)}{\partial y}\right)^{2}\right](\tilde{g} \circ \sigma) d x d y .
\end{aligned}
$$

Therefore, using partitions of unity,

$$
D_{\tilde{X}_{c}}(f)=D_{\tilde{X}_{c}}\left(\sigma^{*}(\tilde{f})\right)<\infty .
$$

From (3) one obtains

$$
\begin{aligned}
D_{\tilde{X}_{c}}\left(\tilde{f}_{1}\right) & =D_{\tilde{X}_{c}}\left(\frac{1}{2} \tilde{f}+\frac{1}{2} \sigma^{*}(\tilde{f})\right) \\
& \leq D_{\tilde{X}_{c}}\left(\frac{1}{2} \tilde{f}\right)+D_{\tilde{X}_{c}}\left(\frac{1}{2} \sigma^{*}(\tilde{f})\right)+2 D_{\tilde{X}_{c}}\left(\frac{1}{2} \tilde{f}, \frac{1}{2} \sigma^{*}(\tilde{f})\right)<\infty
\end{aligned}
$$

since

$$
D_{\tilde{X}_{c}}\left(\frac{1}{2} \tilde{f}, \frac{1}{2} \sigma^{*}(\tilde{f})\right)^{2} \leq D_{\tilde{X}_{c}}\left(\frac{1}{2} \tilde{f}\right) D_{\tilde{X}_{c}}\left(\frac{1}{2} \sigma^{*}(\tilde{f})\right)<\infty .
$$

Finally, from (2) and (4) we conclude that $f_{1}$ has finite Dirichlet integral and thereby $X$ is not in $O_{A D}^{K}$.

6.3. For UNOKS we have the following corresponding analogue to Theorem B.

Theorem 6.2. Let $F$ be an open UNOKS of finite genus $g>2$. Then all the compact UNOKS of genus $g$ which are extensions of $F$ are dianalytically isomorphic to each other if and only if $F$ is in $O_{A D}^{K}$.

Proof. If $F$ is in $O_{A D}^{K}, \tilde{F}_{c}$ is in $O_{A D}$ by Theorem 6.1 and thus, by Theorem A, $P\left(\tilde{F}_{c}\right)$ consists of a single point. But since $i(P(F)) \subset P\left(\tilde{F}_{c}\right)$, we conclude that $i(P(F))$ and $P(F)$ must also consist of a single point, which clearly implies that all the extensions of $F$ are dianalytically isomorphic.

To prove the converse, suppose that all the extensions $W$ of $F$ are dianalytically isomorphic to each other. Then, by lifting the corresponding dianalytic 
isomorphisms, we deduce that all the extensions of $\tilde{F}_{c}$ in $i(P(F))$ are conformally equivalent. This means that $\Pi(i(P(F)))$ consists of a single point, where

$$
\Pi: T_{g-1}\left(\tilde{W}_{0 c}\right) \rightarrow \mathbf{R}_{g-1}\left(\tilde{W}_{0 c}\right)=T_{g-1}\left(\tilde{W}_{0 c}\right) /_{\operatorname{Mod}\left(\tilde{W}_{0 c}\right)}
$$

is the canonical projection of the Teichmüller space onto the Riemann space. By the discontinuity of $\operatorname{Mod}\left(\tilde{W}_{0 c}\right)$ we conclude that $i(P(F))$ must be a discrete set, and since $i(P(F))$ is connected, it must consist of a single point, and so $P(F)$, q.e.d.

\section{References}

[1] Ahlfors, L.V., and L. Sario: Riemann surfaces. - Princeton University Press, Princeton, 1960.

[2] Alling, N.L., and N. Greenleaf: Foundations of the theory of Klein surfaces. - Lecture Notes in Mathematics 219. Springer-Verlag, Berlin-Heidelberg-New York, 1971.

[3] Bochner, S.: Fortsetzung Riemannscher Flächen. - Math. Ann. 98, 1927, 406-421.

[4] Fernandez, A.: On extensions of Klein surfaces. - Preprint.

[5] Jussila, T.: On extensions of Riemann surfaces. - Ann. Acad. Sci. Fenn. Ser. A I Math. Dissertationes 67, 1988, 1-36.

[6] Leнто, O.: Univalent functions and Teichmüller spaces. - Graduate Texts in Mathematics. Springer-Verlag, Berlin-Heidelberg-New York, 1987.

[7] Macbeath, A.M., and D. Singerman: Spaces of subgroups and Teichmüller space. Proc. London Math. Soc. (3) 31, 1975, 211-256.

[8] Massey, W.: Algebraic topology: An introduction. - Harcourt, Brace \& World Inc., New York, 1967.

[9] OikaWA, K.: On the prolongation of an open Riemann surface of finite genus. - Kodai Math. Sem. Rep. 9, 1957, 34-41.

[10] OikaWA, K.: On the uniqueness of the prolongation of an open Riemann surface of finite genus. - Proc. Amer. Math. Soc. 11, 1960, 785-787.

[11] SeppäLё, M.: Teichmüller spaces of Klein surfaces. - Ann. Acad. Sci. Fenn. Ser. A I Math. Dissertationes 15, 1978, 1-37.

[12] Timman, S.: Einbettungen endlicher Riemannscher Flächen. - Math. Ann. 217, 1975, 8185.

[13] Zieschang, H., E. Vogt, and H.D. Coldewey: Surfaces and planar discontinuous groups. - Lecture Notes in Mathematics 835. Springer-Verlag, Berlin-HeidelbergNew York, 1980.

A.J. Alonso Gomez

Universidad Nacional de Educacion a Distancia

Department of Mathematics

Faculty of Science

Madrid

Spain

Received 2 March 1989

\section{A. Fernandez Arias}

Universidad Nacional de Educacion a Distancia

Department of Mathematics

Faculty of Science

Madrid

Spain 